\title{
Hematopoietic lineage-converted T cells carrying tumor-associated antigen- recognizing TCRs effectively kill tumor cells
}

To cite: Hu F, Huang D, Luo Y, et al. Hematopoietic lineageconverted T cells carrying tumor-associated antigenrecognizing TCRs effectively kill tumor cells. Journal for ImmunoTherapy of Cancer 2020;8:e000498. doi:10.1136/ jitc-2019-000498

- Additional material is published online only. To view please visit the journal online (http://dx.doi.org/10.1136/jitc2019-000498).

$\mathrm{FH}$ and $\mathrm{DH}$ contributed equally.

$\mathrm{FH}$ and $\mathrm{DH}$ are joint first authors.

Accepted 16 June 2020

\section{Check for updates}

(C) Author(s) (or their employer(s)) 2020. Re-use permitted under CC BY. Published by BMJ.

For numbered affiliations see end of article.

\section{Correspondence to} Dr Jinyong Wang; wang_jinyong@gibh.ac.cn

Dr Hongling Wu; wu_hongling@gibh.ac.cn

\section{ABSTRACT}

Tumor-associated antigen (TAA) T-cell receptor (TCR) gene-engineered $\mathrm{T}$ cells exhibit great potential in antitumor immunotherapy. Considering the high costs and low availability of patient-derived peripheral blood T cells, substantial efforts have been made to explore alternatives to natural $T$ cells. We previously reported that enforced expression of Hoxb5 converted B cells into induced T (iT) cells in vivo. Here, we successfully regenerated naive 0T1 (major histocompatibility complex I restricted ovalbumin antigen) iT cells (0T1-iT) in vivo by expressing Hoxb5 in pro-pre-B cells in the 0T1 transgenic mouse. The 0T1iT cells can be activated and expanded in vitro in the presence of tumor cells. Particularly, these regenerated OT1-iT cells effectively eradicated tumor cells expressing the TAA (ovalbumin) both in vitro and in vivo. This study provides insights into the translational applications of blood lineage-transdifferentiated $T$ cells in immunotherapy.

\section{INTRODUCTION}

Tumor-associated antigen (TAA) T-cell receptor (TCR) gene-engineered T cell (TAATCR-T) therapy has shown great prospect in treating malignant cancers such as melanoma, sarcoma, mesothelioma, and other malignancies. ${ }^{12}$ Numerous research groups have been focusing on preparing high-avidity TCRs of TAA, and maintaining the T-cell activity and longevity in vitro during stimulation and expansion. ${ }^{3-5}$ In regenerative medicine, it has been a central aim to produce cellular alternatives to natural peripheral blood (PB) T cells. One conventional attempt is to deliver tumor-specific TCR genes into the hematopoietic stem cells (HSCs), which can differentiate into antitumor T cells. ${ }^{6}{ }^{7}$ However, this approach contains the risk of a patient sustainably producing TAA-TCR-T cells throughout their lifespan, as well as the potential contamination of TCR expression in other blood lineage cells. Recently, scientists have turned their emphasis on induced pluripotent stem cells (iPSCs), as TAA-TCRs can be introduced into iPSCs to form TAATCRs-iPSC clones without compromising the key traits of these stem cells. ${ }^{8-10}$ Nonetheless, a fast method of regenerating TAA-TCR in vivo remains elusive.

Blood lineages can be regenerated by direct lineage transdifferentiation approaches. ${ }^{1-14}$ Recently, we reported that B cells can be converted into functional $\mathrm{T}$ cells by Hoxb5 protein, a transcription factor that is not expressed in B cells nor in T cells. ${ }^{15}$ Here, we translationally extended our study and regenerated TAA-TCR induced $\mathrm{T}$ (iT) cells by manipulating the OT1 pro-pre-B cells sorted from the OT1 transgenic mouse using a retrovirus delivery system expressing the Hoxb5 in vivo. Major histocompatibility complex I (MHC-I) restricted CD8 ${ }^{+}$OT1-iT cells were successfully regenerated in the peripheral immune organs of the recombination activating gene 1 mutation $\left(\operatorname{Rag} 1^{--}\right)$recipients, a mouse strain lacking natural $\mathrm{T}$ and $\mathrm{B}$ cells. In vitro and in vivo functional assays provide robust evidence that the regenerated TAATCR-iT cells have the capacity of specifically killing tumor cells expressing the TAA. Regarding the short-time window, transiency, perfect development of iT regeneration process in vivo by B-to-T lineage transdifferentiation, ${ }^{15}$ we document a de novo alternative approach to regenerate TAA-TCR iT cells by blood lineage transdifferentiation in vivo.

\section{RESULTS}

Ectopic expression of the Hoxb5 reprogrammed OT1 B cells into OT1-iT cells

To produce OT1-iT cells converted from the OT1 pro-pre-B cells, we sorted OT1 pro-pre-B cells 
A

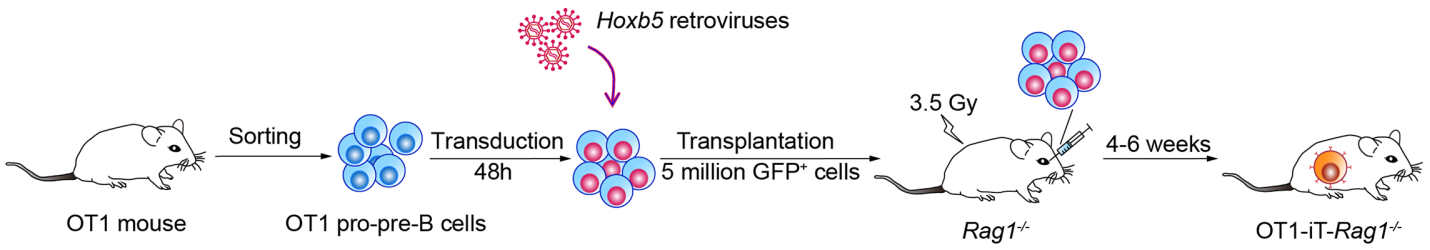

B

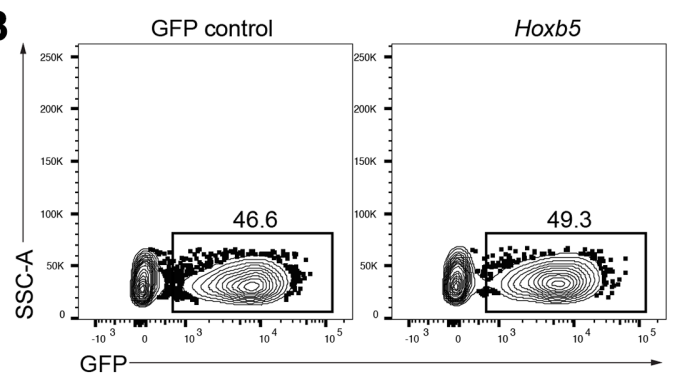

D

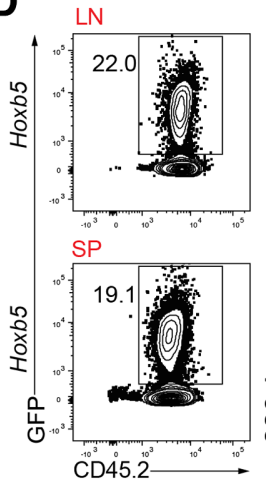

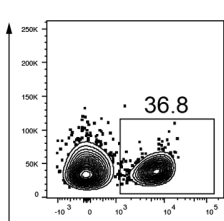

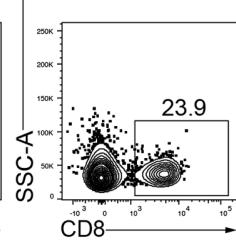

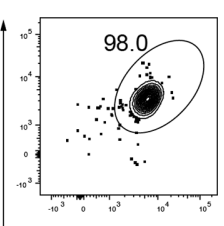

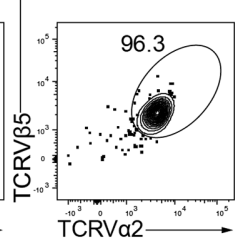

C
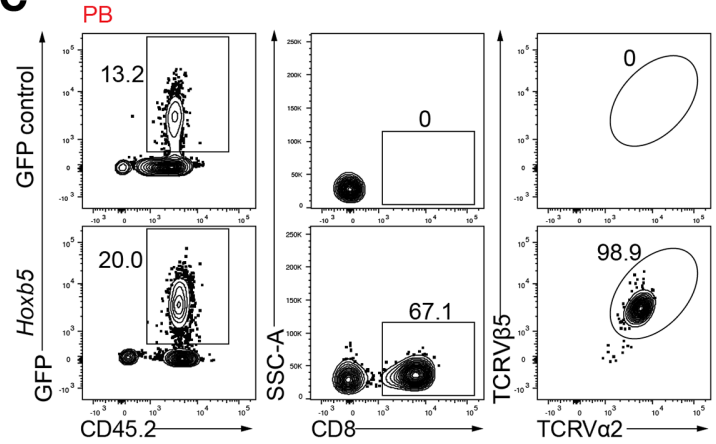

$\mathbf{E}$

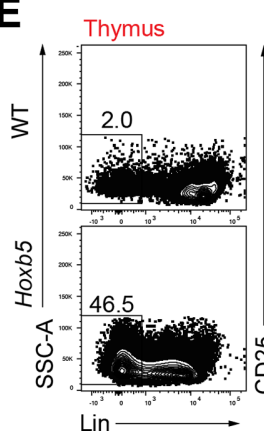

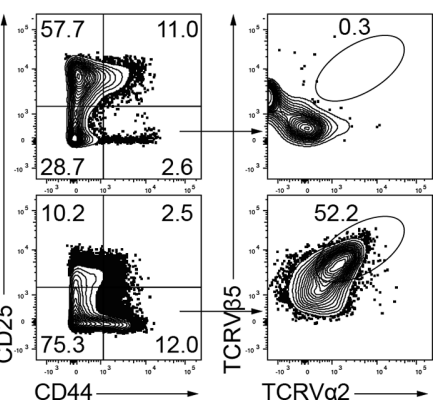

Figure 1 Immunophenotypic characterization of the OT1-iT cells. (A) Schematic strategy of generating OT1-iT by ectopic expression of Hoxb5 retroviruses in OT1 pro-pre-B cells. OT1 pro-pre-B cells were sorted from bone marrow-nucleated cells from OT1 transgenic mouse (C57BL/6 mouse strain), transduced with the Hoxb5 retroviruses, and subsequently transplanted into irradiated Rag $1^{-/-}$mice (3.5 Gy, 5 million GFP ${ }^{+}$cells per mouse, OT1-iT-Rag $1^{-/}$). (B) The transduction rates of the OT1 propre-B cells infected with either the Hoxb5 or GFP control retroviruses. Hoxb5 retroviruses or GFP control retroviruses were transduced into the OT1 pro-pre-B cells (GFP control or Hoxb5) by two rounds of spin transfection. The GFP-positive population indicated the infected OT1 pro-pre-B cells. (C) Flow cytometric analysis of the mature OT1 iT cells in the PB of the OT1-iTRag $1^{-/-}$mouse 4 weeks' post-transplantation. OT1-positive iT cells were defined as CD $45.2^{+} \mathrm{GFP}^{+} \mathrm{CD} 8^{+} \mathrm{TCRV} \alpha 2^{+} \mathrm{TCRV} \beta 5^{+}$. Representative plots from recipients of GFP control OT1 pro-pre-B (GFP control) and Hoxb5 OT1 pro-pre-B (Hoxb5) are shown. (D) Flow cytometric analysis of the mature OT1 iT cells in the LN and SP of the OT1-iT-Rag $1^{-1-}$ mice (Hoxb5) 4 weeks' posttransplantation. (E) Intracellular staining of the expression of the OT1 in the donor-derived thymocytes. DN1 cells with the

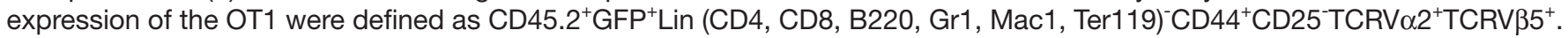
Flow plots of one representative recipient (Hoxb5) and WT control mouse are shown. SSC-A, side scatter area; DN1, stage 1 double-negative thymocytes; iT, induced T cells; LN, lymph nodes; PB, peripheral blood; SP, spleen; TCR, T-cell receptor; WT, wild type.

(CD3 Mac1 Ter119-B220 ${ }^{+} \mathrm{CD} 19^{+} \mathrm{CD}^{-} 3^{+} \mathrm{IgM}^{-}$) from the bone marrow nucleated cells of OT1 C57BL/6 transgenic mice and transduced them with Hoxb5 retroviruses or green fluorescent protein (GFP) control following a previous protocol. ${ }^{16}$ Next, the transduced cells were retro-orbitally transplanted into sublethally irradiated Rag $1^{-1}$ mice (C57BL/6, $3.5 \mathrm{~Gy}, 5$ million cells/mouse) to generate the OT1-iT cells (online supplementary figure S1a; figure 1A,B). Four to six weeks post-transplantation, the OT1-iT cells appeared in the PB, lymph node (LN), and spleen (SP) of the recipient OT1-iT-Rag1 ${ }^{-1-}$ mice (figure 1C,D). Additionally, the OT1-TCR proteins were expressed on the surface of the stage 1 doublenegative thymocytes (DN1 cells) in the thymus of the
OT1-iT-Rag $1^{--}$mice (figure 1E). As expected, there were no iT generated in the $\mathrm{PB}$ of the $\mathrm{Rag} \mathrm{1}^{-/-}$recipients transplanted with GFP control transduced pro-pre-B cells (figure 1C). To validate that the OT1-iT cells were derived from the OT1 pro-pre-B cells rather than natural OT1 T-cell contaminants, we performed DNA sequencing of B cell receptor (BCR) heavy chain ( $\mathrm{IgH})$ rearrangements using the genome from the single OT1-iT cells which were sorted from the SP of the OT1-iT-Rag ${ }^{-/}$mouse using a previously reported protocol. ${ }^{15}$ As expected, the single OT1-iT cells contained B-cell antigen receptor immunoglobulin heavy-chain $\mathrm{V}(\mathrm{D}) \mathrm{J}$ rearrangements (online supplementary figure S1b), which signaled their B cell origin. Furthermore, donor-derived $\mathrm{LinS}^{-} \mathrm{Scal}^{+} \mathrm{C}^{\mathrm{k}} \mathrm{kit}^{+}$(LSK) 

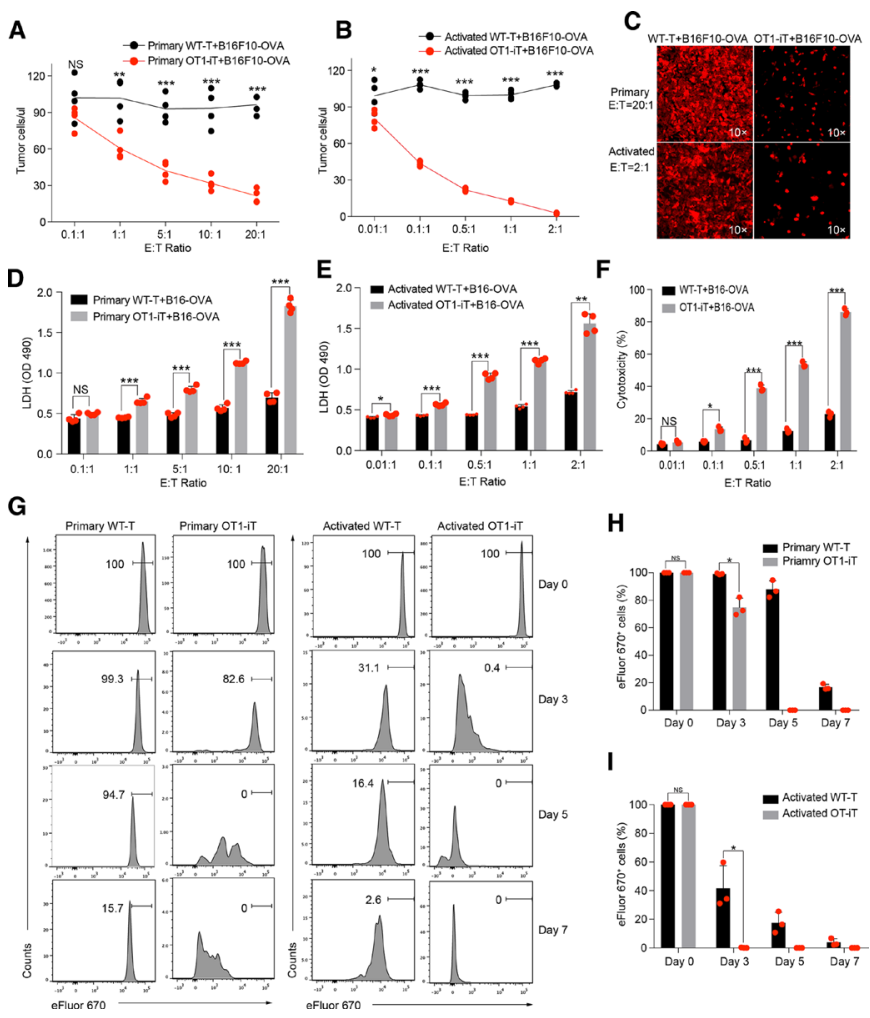

Figure 2 OT1-iT cells exhibit antitumor activity in vitro. Primary OT1-iT cells were enriched from the spleens of the OT1-iTRag $1^{-1 /}$ mice 6 weeks' post-transplantation. Activated OT1-iT cells were obtained by stimulating primary OT1-iT cells with antiCD3/CD28 beads for 4 days. (A) Cell number of the B16F10-OVA tumor cells after 36 hours of coculture with primary OT1-iT cells. A total of $1 \times 10^{4}$ B16F10-OVA tumor cells as target $(\mathrm{T})$ cells were cocultured with primary OT1-iT $\left(1 \times 10^{3}, 1 \times 10^{4}, 5 \times 10^{4}\right.$, $1 \times 10^{5}$, and $2 \times 10^{5}$ ) or control WT-T cells as effector (E) cells for 36 hours at different E:T ratios $(0.1: 1,1: 1,5: 1,10: 1,20: 1)(n=4)$. (B) The number of B16F10-OVA tumor cells after 36 hours co-culture with activated OT1-iT cells. A total of $1 \times 10^{4}$ B16F10-OVA tumor cells as target (T) cells were co-cultured with activated OT1-iT $\left(1 \times 10^{2}, 1 \times 10^{3}, 5 \times 10^{3}, 1 \times 10^{4}\right.$, and $\left.2 \times 10^{4}\right)$ as effector $(\mathrm{E})$ cells for 36 hours at different E:T ratios $(0.01: 1,0.1: 1,0.5: 1,1: 1,2: 1)(n=4)$. (C) Representative microphotograph of primary and activated OT1-iT cells cocultured with B16F10-OVA tumor cells at the indicated E:T ratio for 36 hours. (D) LDH release of the B16F10-OVA tumor cells after 36 hours' coculture with primary OT1-iT cells or control WT-T cells $(n=4)$. (E) LDH release of the B16F10-OVA tumor cells after 24 hours' coculture with activated OT1-iT cells or control WT-T cells ( $n=4)$. (F) Cytotoxicity of the OT1-iT cells or control WT-T cells cocultured with B16F10-OVA tumor cells for 24 hours $(n=3)$. (G) Proliferation of the primary and activated OT1-iT and control WT-T cells responding to B16F10-OVA stimulation in vitro. The primary and activated OT1-iT cells or control WT-T cells $\left(1 \times 10^{5}\right)$ labeled with cell proliferation dye eFluor 670 were stimulated with $1 \times 10^{5}$ B16F10-OVA tumor cells, and the proliferation status of the OT1-iT cells or WT-T cells were analyzed after coculture at day 3, day 5, and day 7 . (H) Statistical analysis of $\mathrm{CD}^{+}$eFluor670 $0^{+}$primary OT1-iT cells or control WT-T cells in (G), left $(n=3)$. (I) Statistical analysis of CD8 ${ }^{+}$eFluor670 ${ }^{+}$-activated OT1-iT cells or control WT-T cells in $(G)$, right $(n=3)$. Data are representative of three independent experiments and were analyzed by two-sided independent $t$-test (A, B, D, E, F, H, and I) or Mann-Whitney test (D). ${ }^{*} \mathrm{p}<0.05$, ${ }^{* *} \mathrm{p}<0.01,{ }^{* * *} \mathrm{p}<0.001$. iT, induced T cells; LDH, lactate dehydrogenase; NS, not significant; OD, optical density; OVA, ovalbumin; WT, wild type.

and common lymphoid progenitor (CLP) cells were absent in the bone marrow of the recipients' 6 weeks' post-transplantation (online supplementary figure S1c), which further excludes the possibility of donor long-term HSC contamination. Collectively, these results indicate that OT1 pro-pre-B cells can be converted into OT1-iT cells in the presence of Hoxb5.

\section{OT1-iT cells specifically kill B16F10-OVA tumor cells in vitro}

To establish the tumor targets of the OT1-iT cells, we constructed an ovalbumin (OVA)-expressing B16F10 melanoma cell line (B16F10-OVA), which presents the MHC-I restricted OVA antigen. Next, we cocultured SP-derived OT1-iT cells (effector (E) cell) with
B16F10-OVA cells (target $(\mathrm{T})$ cell) to examine their antitumor activity. We chose wild-type T cells (WT-T) from C57BL/6 mouse as the negative control for their natural TCR repertoire diversity. Particularly, primary splenic OT1-iT cells $\left(1 \times 10^{3}, 1 \times 10^{4}, 5 \times 10^{4}, 1 \times 10^{5}\right.$, and $\left.2 \times 10^{5}\right)$ or splenic WT-T cells $\left(1 \times 10^{3}, 1 \times 10^{4}, 5 \times 10^{4}, 1 \times 10^{5}\right.$, and $2 \times 10^{5}$ ) isolated from OT1-iT-Rag $1^{--}$or WT mice were cocultured with $1 \times 10^{4}$ B16F10-OVA cells. The number of B16F10-OVA cells sharply decreased after 36 hours of coculture with the primary OT1-iT cells at various E:T ratios (figure 2A,C upper panel and figure 2D) compared with the WT-T control group $(\mathrm{p}<0.01, \mathrm{p}<0.001)$. Moreover, $\mathrm{CD} 8^{+}$OT1-iT cells exhibited robust proliferation in 


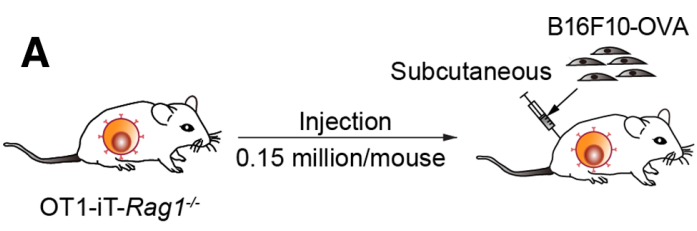

B
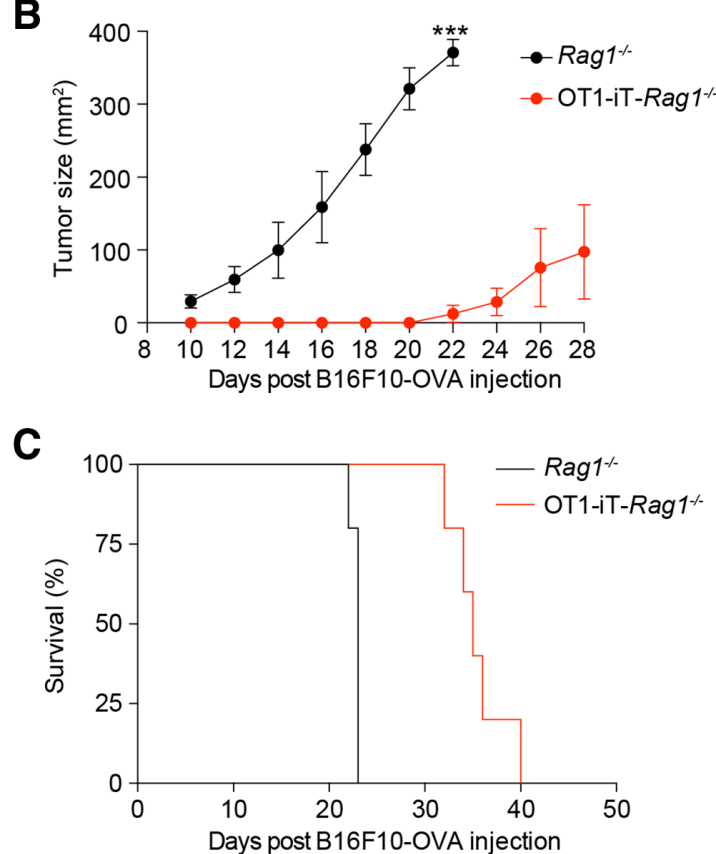

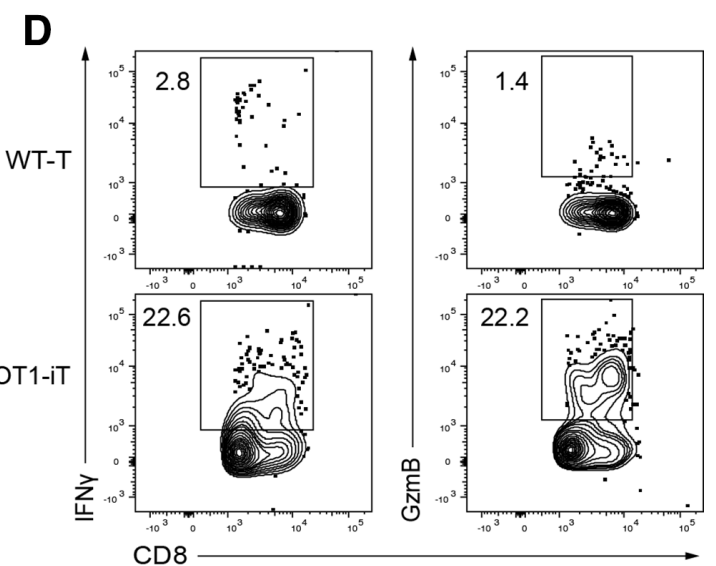

$\mathbf{E}$

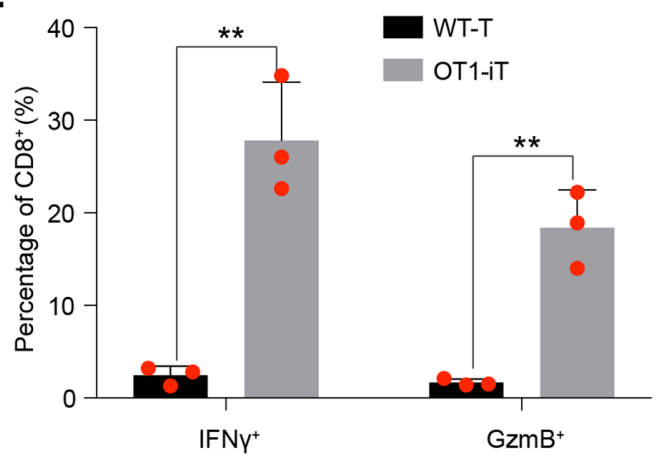

Figure 3 Prevention of the B16F10-OVA tumor cell growth in OT1-iT-Rag1/- mice. (A) Schematic diagram of the primary OT1iT cells for anti-tumor therapy in OT1-iT-Rag $1^{-/-}$mice. B16F10-OVA cells $(0.15$ million/mouse) were subcutaneously injected into the groin of the Rag $1^{-/-}$or OT1-iT-Rag $1^{-/-}$mice 6 weeks after transplantation with OT1 pro-pre-B cells transduced with Hoxb5 retroviruses. (B) Tumor growth in Rag1/- (control) and OT1-iT- Rag ${ }^{-/-}$mice (OT1-iT). The tumor sizes (length $\times$width, $\mathrm{mm}^{2}$ ) were measured using a caliper every other day. Mice with tumor size larger than $400 \mathrm{~mm}^{2}$ were euthanized following ethical standard (each group $n=5)$. (C) Kaplan-Meier survival curve of Rag1/- (control) or OT1-iT-Rag $1^{-/-}$mice post-B16F10-OVA injection $(n=5$, $\mathrm{p}=0.0018$, log-rank test). (D) Intracellular staining of IFN $\gamma$ and GzmB in the infiltrated OT1-iT cells. T cells were isolated from the OT1-iT-Rag $1^{-1-}$ mouse when tumor size reached $400 \mathrm{~mm}^{2}$. The IFN $\gamma^{+}$or $\mathrm{GzmB}^{+}$population is shown for the CD45.2 $\mathrm{Mac1}^{-} \mathrm{Gr} 1^{-}$ $\mathrm{CD}^{+}$iT cells. The bone marrow from wild-type (WT) mouse was used as the negative control to define the positive population of IFN $\gamma^{+}$or GzmB. Flow plots from one representative mouse of each group are shown. (E) Percentage of IFN $\gamma^{+}$or $\mathrm{GzmB}^{+} \mathrm{CDB}^{+}$ OT1-iT cells and WT-T cells in (D) $(n=3)$. Data are representative of three independent experiments and were analyzed by twosided-independent $t$-test (B, E). ${ }^{* *} \mathrm{p}<0.01,{ }^{* \star *} \mathrm{p}<0.001$. GzmB, granzyme B; IFN $\gamma$, interferon gamma; iT, induced T cells; OVA, ovalbumin; WT, wild type.

the presence of the B16F10-OVA cells within 7 days, while control CD8 ${ }^{+}$WT-T cells proliferated much more slowly. This indicated direct tumor cell-stimulated activation of the OT1-iT cells (figure 2G, left panel and figure $2 \mathrm{H}$ ). To test whether T-cell activation preceding the tumor cell coculture could enhance the tumor-killing ability of the OT1-iT cells, we stimulated the primary OT1-iT cells or the primary WT-T cells with CD3/CD28 antibodies for 4 days in vitro. Strikingly, a much lower number of the preactivated OT1-iT cells $\left(1 \times 10^{2}, 1 \times 10^{3}, 5 \times 10^{3}, 1 \times 10^{4}\right.$, and $2 \times 10^{4}$ ) than the primary OT1-iT cells could significantly kill the B16F10-OVA cells $\left(1 \times 10^{4}\right)$ in vitro, whereas the activated WT-T cells still exhibited no-killing behavior even at the highest $\mathrm{E}$ :T ratio $(2: 1)(\mathrm{p}<0.01, \mathrm{p}<0.001)$ (figure 2B,C lower panel and figure $2 \mathrm{E}$ ). The proliferation experiments also showed that the activated OT1-iT cells also proliferated much faster than the activated WT-T cells after coculture with the B16F10-OVA cells (figure 2G, right panel and figure 2I). Moreover, the OT1-iT cells demonstrated significantly efficient cytotoxicity activity over WT-T cells as the E:T ratio increased (figure 2F), and the proliferation of the cocultured B16F10-OVA tumor cells was inhibited in the OT1-iT group $(\mathrm{p}<0.5)$ (online supplementary figure S2a and b). Taken together, the OT1-iT cells converted from B cells effectively killed tumor cells in vitro.

\section{T1-iT cells suppress tumor growth in vivo}

To evaluate the impact of OT1-iT cells on B16F10-OVA tumor proliferation in OT1-iT-Rag ${ }^{-1-}$ mice, B16F10-OVA tumor cells were subcutaneously injected into OT1-iTRag ${ }^{-1}$ mice 6 weeks' post-transplantation (figure 3A). Consequently, the tumor sizes were less than $160 \mathrm{~mm}^{2}$ on day 28 post-B16F10-OVA injection, while those in the 
control ( Rag $1^{-/}$transplanted with B16F10-OVA) group were around $400 \mathrm{~mm}^{2}$ on day 22 postinjection, leading to euthanasia to comply with experimental animal ethics procedures (figure 3B). Comparatively, the B16F10-OVA tumor-bearing OT1-iT-Rag $T^{-}$mice survived up to 40 days (figure 3C), demonstrating a prolonged survival than the untreated control. In addition, we observed that the tumor-infiltrated OT1-iT cells $\left(\mathrm{CD} 45.2^{+} \mathrm{CD} 8^{+}\right)$ secreted interferon gamma (IFN $\gamma$ ) and granzyme B (GzmB) (figure 3D,E), which indicated their tumor cell killing behavior. Thus, these results demonstrated that the OT1-iT cells can reduce tumor development in OT1-iT-Rag $1^{-/}$mouse.

To mimic the treatment scenario of patients with tumor, we performed an adoptive transfer assay of the SP-derived OT1-T cells (OT1 transgenic mouse, positive control) and OT1-iT cells to allogenic mice bearing tumors. We isolated splenic OT1-T cells from OT1 mice, OT1-iT cells from primary OT1-iT-Rag $1^{-1}$ mice, and WT-T cells from the WT mice followed by expansion and activation, and then adoptively transferred them into tumor-bearing mice (figure 4A). As expected, the tumor burden in the OT1-iT cell-treated B16F10-OVA tumor-bearing mice was significantly reduced, and these mice survived up to 43 days post-B16F10-OVA tumor cell injection. This achieved comparable therapeutic effects as the OT1-T cell treatment (up to 42 days). In contrast, tumor sizes of WT-T cell-treated mice reached the limit of ethic allowance within 28 days' postinjection, resulting in their sacrifice (figure 4B,C). We further analyzed the activation status of the OT1-iT and OT1-T cells infiltrated in the tumors and observed that both the OT1-iT and OT1-T cells $\left(\mathrm{CD} 45.2^{+} \mathrm{CD} 8^{+}\right)$were completely activated $\left(\mathrm{CD} 44^{\text {hi }} \mathrm{C}-\right.$ D69 $\left.{ }^{+} \mathrm{CD} 62 \mathrm{~L}^{-}\right)$, comparing with primary $\mathrm{T}$ cells. Notably, OT1-iT cells had more activated $\mathrm{CD} 44^{\mathrm{hi}} \mathrm{CD} 69^{+} \mathrm{CD} 62 \mathrm{~L}^{-}$ cells than OT1-T cells (figure 4D,E). In addition, the activated OT1-iT cells secreted comparable levels of IFN $\gamma$ and GzmB to OT1-T cells (figure 4F,G), indexing their tumor-eradicating behavior. Furthermore, programmed cell death protein 1 (PD-1) expression was upregulated in the infiltrated OT1-T and OT1-iT cells in the mice with tumor recurrence (figure $4 \mathrm{H}, \mathrm{I}$ ). These results illustrate the antitumor capacity of the reprogrammed OT1-iT cells in vivo.

\section{DISCUSSION}

In this study, we generated naive OT1-iT cells in $\operatorname{Rag}^{-1-}$ mouse from Hoxb5-overexpressing pro-pre-B cells. Just as previously reported,${ }^{15}$ it takes 4 weeks to obtain OT1-iT cells in recipients transplanted with Hoxb5-overexpressing pro-pre-B cells, which is much shorter than either HSCderived or iPSC-derived OT1-iT cells (7-8 weeks). ${ }^{6} 10$ However, the starting pro-pre-B cells in our study were collected from bone marrow, which is an obstacle for further translational research since preparing bone marrow-derived cells were invasive and quantity limited. Thus, new methods need to be developed to obtain abundant pro-pre-B cells in vitro, such as via natural hematopoietic stem and progenitor cells (HSPC) differentiation and expansion. ${ }^{1718}$

Besides the Hoxb5-expressing OT1 pro-pre-B cells can generate OT1-iT cells, the WT pro-pre-B cells without expressing OT1 can also transdifferentiate into OT1-iT cells when simultaneously enforcing expression of Hoxb5 and OT1 TCR (online supplementary figure S3a-c). Moreover, these OT1-iT cells also significantly reduced the tumor burden and prolonged survival (online supplementary figure S3d-e). Of note, this tandem expression approach showed much lower assembling efficiencies of OT1 TCR $\alpha \beta$ chains in the $\mathrm{CD} 8^{+}$iT cells than by OT1 transgenic method, which is largely due to the optimized construction strategy of the OT1 transgenic mouse. ${ }^{19}$ In addition, endogenous TCR $\alpha$ chains can also have additional rearrangements in the presence of exogenous ones since TCR $\alpha$ loci rearrangements have no allelic exclusion phenomenon. ${ }^{20}{ }^{21}$ Expectedly, it can improve the efficiency of OT1-iT cells by searching for a stronger promoter or enhancer to competitively express OT1 TCR $\alpha$ chains or directly blocking additional endogenous TCR rearrangements by knockdown of RAG recombinase expression.

We have confirmed that the transdifferentiationderived OT1-iT cells can prevent tumor growth both in reconstituted OT1-iT-Rag $1^{-/}$and adoptive tumor-bearing models. Alternatively, it is worth trying to directly generate OT1-iT cells in tumor models to evaluate their antitumor ability, as this way mimics natural disease development. In conclusion, we have developed an alternative method of generating tumor-specific iT cells in animals by a de novo blood lineage-transdifferentiation approach.

\section{MATERIALS AND METHODS}

\section{Generation and analysis of the 0T1-iT cells}

Pro-pre-B cells' (C57BL/6 mouse) or OT1 pro-pre-B cells' (OT1 transgenic mouse) isolation, infection, and transfer were performed as previously described. ${ }^{16}$ Briefly, propre-B cells from WT mice or OT1 transgenic mice were first enriched via positive magnetic affinity cell sorter selection using B220-biotin and anti-biotin MicroBeads (Miltenyi Biotec), and then sorted from the enriched $\mathrm{B}_{220}{ }^{+}$cells by Aria III (BD). The sorted cells were subsequently stimulated with the pro-pre-B cell medium for 12-16 hours prior to retroviral transduction. Pro-pre-B cells (Hoxb5 retrovirus or OT1-Hoxb5 retrovirus) and OT1 pro-pre-B cells (GFP retrovirus or Hoxb5 retrovirus) were transduced with retrovirus by two rounds of spin transfection $\left(800 \mathrm{~g}, 90 \mathrm{~min}, 35^{\circ} \mathrm{C}\right)$ at a density of 1 million/ $\mathrm{mL}$. For transplantation, 5 million $\mathrm{GFP}^{+}$pro-pre-B cells or OT1 pro-pre-B cells were injected into the retro-orbital veins of the irradiated Rag ${ }^{-1-}$ recipients $(3.5 \mathrm{~Gy}, \mathrm{RS} 2000$; $\mathrm{Rad}$ Source). All recipients were given water supplemented with trimethoprim-sulfamethoxazole for 2 weeks to prevent infection. OT1-iT lymphocytes were analyzed 4-6 weeks' post-transplantation. 


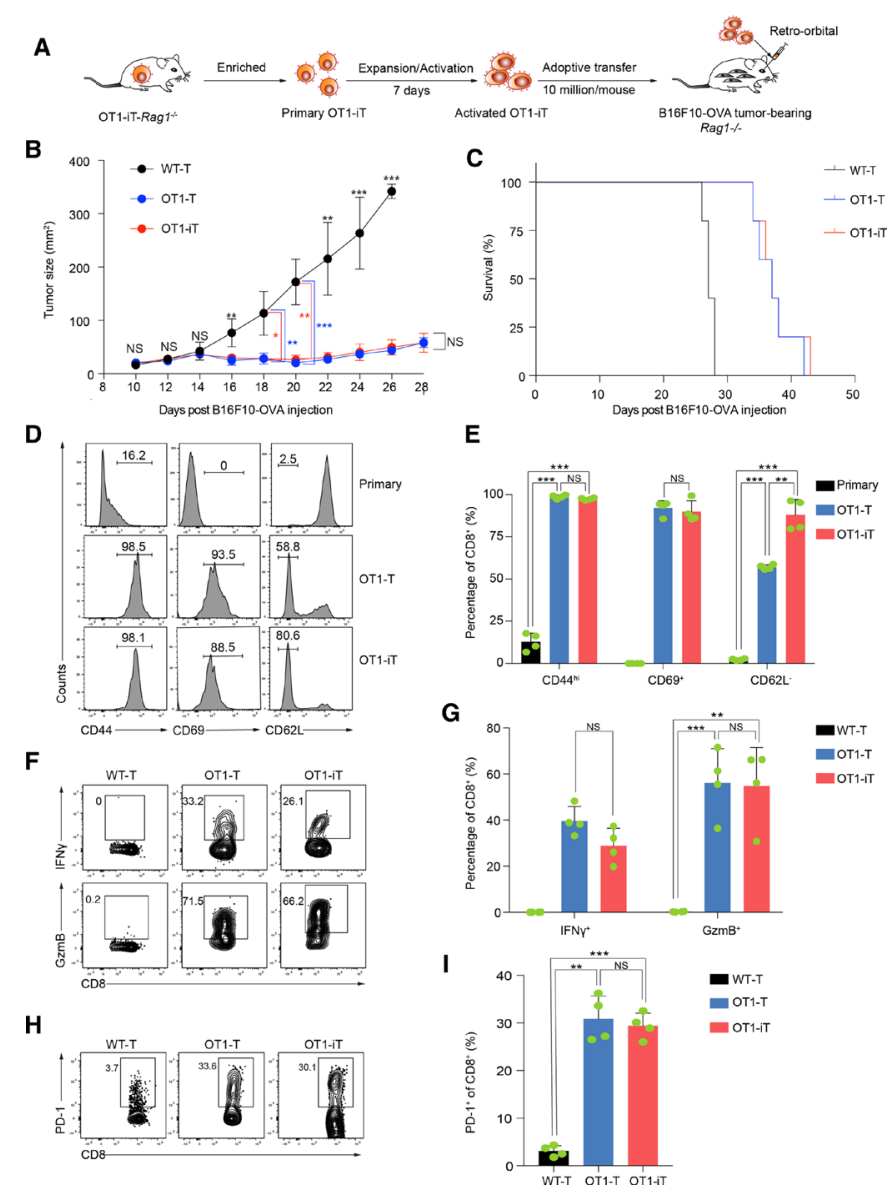

Figure 4 Adoptive transfer of OT1-iT cells relieves the tumor burden in vivo. (A) Schematic diagram of the OT1-iT cells for antitumor therapy in the B16F10-OVA tumor-bearing Rag $1^{1 /-}$ mice. Rag $1^{-/-}$mice were subcutaneously injected with B16F10OVA cells ( 0.15 million/mouse) in the groin to establish the melanoma tumor model. Expanded and activated OT1-iT cells were obtained by stimulating primary OT1-iT cells with anti-CD3/CD28 beads for 7 days. The activated OT1-iT cells (10 million/ mouse) were transplanted into the tumor-bearing mice 10 days after the tumor cell injections. (B) Tumor growth in B16F10OVA tumor-bearing Rag $1^{-1-}$ mice. The tumor-bearing mice with similar tumor size were randomly divided into three groups and received activated WT-T, OT1-T, or OT1-iT cells (10 million/mouse) 10 days after B16F10-OVA tumor cell injection ( $\mathrm{n}=5$ each group). The tumor sizes (length $\times$ width, $\mathrm{mm}^{2}$ ) were measured using a caliper every other day. Tumor size data are shown from day 10 to day 28. Mice with tumor sizes larger than $400 \mathrm{~mm}^{2}$ were euthanized for ethical consideration. (C) Kaplan-Meier survival curve of the tumor-bearing Rag $1^{1 / 2}$ mouse ( $n=5$ each group, $p=0.0002$, log-rank test). (D) The activation status of the tumor-infiltrating OT1-T and OT1-iT cells. Tumor-bearing Rag $1^{-1-}$ mice transplanted with the T cells were sacrificed when the tumor size reached $400 \mathrm{~mm}^{2}$, and the tumor-infiltrating T cells were isolated from the tumors for further flow cytometric analysis. Flow plots of the $\mathrm{CD} 44^{\mathrm{hi}}, \mathrm{CD}^{+} 9^{+}$, and $\mathrm{CD} 2 \mathrm{~L}^{-}$population of one representative mouse from each group are shown for the gated CD 45. $2^{+} \mathrm{Mac1}^{-} \mathrm{Gr}^{-} \mathrm{CD}^{+} \mathrm{T}$ cells. Primary T cells isolated from the peripheral blood of OT1-iT-Rag $1^{-/-}$mice were used as negative control. (E) Percentage of CD44 ${ }^{\mathrm{hi}}$, CD69 ${ }^{+}$and CD62L' cells in (D) $(n=4)$. (F) Intracellular staining of IFN $\gamma$ and $\mathrm{GzmB}$ in the tumorinfiltrating OT1-T and OT1-iT cells from treated tumor-bearing Rag $1^{-1 /}$ mice. WT-T cells isolated from the bone marrow of WT mice were used as control. (G) Percentage of IFN $\gamma^{+}$and $\mathrm{GzmB}^{+}$populations of WT-T cells, tumor-infiltrating OT1-T, and OT1-iT cells in ( $F)(n=4)$. (H) Flow cytometric analysis of PD-1 of WT-T cells, tumor-infiltrating OT1-T, and OT1-iT cells. (I) Percentage of $\mathrm{PD}-1^{+}$cells in $(\mathrm{H})$. Data are representative of three independent experiments and were analyzed by two-sided independent $t$-test (B, E, G, and I) or Mann-Whitney test (E). ${ }^{*} \mathrm{p}<0.05,{ }^{* \star} \mathrm{p}<0.01,{ }^{* \star *} \mathrm{p}<0.001$. GzmB, granzyme B; IFN $\gamma$, interferon gamma; iT, induced T cells; NS, not significant; OVA, ovalbumin; WT, wild type.

\section{In vitro function analysis of the OT1-iT cells}

Primary OT1-iT cells or WT-T cells derived from the SP of the OT1-iT-Rag $1^{-/-}$mice or C57BL/6 mice were enriched by depletion of Ter $119^{+} \mathrm{CD} 11 \mathrm{~b}^{+} \mathrm{Gr} 1^{+} \mathrm{B} 220^{+} \mathrm{NK} 1.1^{+} \mathrm{CD} 11 \mathrm{c}^{+}$ cells and cultured in the T-cell medium without interleukin 2 at a density of $1 \times 10^{6} / \mathrm{mL}$. Activated OT-iT cells were obtained by coculturing primary OT1-iT cells with Dynabeads Mouse T-Activator CD3/CD28 (CD3/CD28 Gibco) for T-cell expansion and activation for 4 days.
Primary or activated OT1-iT (E) cells were incubated with $1 \times 10^{4}$ B16F10-OVA $(\mathrm{T})$ cells in 96-well plates for 36 hours at respective $\mathrm{E}: \mathrm{T}$ ratios (primary $\mathrm{T}$ cells, $\mathrm{E}: \mathrm{T}=0.1: 1$, 1:1, 5:1, 10:1, 20:1; activated $\mathrm{T}$ cells, $\mathrm{E}: \mathrm{T}=0.01: 1,0.1: 1$, $0.5: 1,1: 1,2: 1)$. The number of B16F10-OVA tumor cells $\left(\right.$ DsRed $\left.^{+}\right)$were enumerated using the CountBright Absolute Counting Beads (Thermo Fisher) by LSRFortessa-X20 (BD). Microphotographs were taken to assess the antitumor effect of primary or activated OT1-iT using the 
ImageXpress Micro Confocal (Molecular Devices). The Non-Radioactive Cytotoxicity Assay kit (Promega) was used to analyze the lactate dehydrogenase released from the coculture cells, and the cytotoxicity of the OT1-iT cells were analyzed following the instructions of the assay kit.

\section{In vitro $\mathrm{OT}$-iT cell proliferation assay}

The proliferation analysis of the OT1-iT cells was performed as described. ${ }^{22}$ Prior to coculture, OT1-iT cells or WT-T cells were stained with the Cell Proliferation Dye eFluor 670 according to the manufacturer's directions. The stained $\mathrm{T}$ cells and B16F10-OVA were cocultured with T-cell medium. The proliferation status of the OT1-iT cells $\left(\mathrm{CD} 45.2^{+} \mathrm{CD}^{+}\right)$were analyzed at day 0 , day 3 and day 7 after coculture by the LSRFortessa-X20 (BD).

\section{B16F10-0VA melanoma tumor model}

OT1-iT-Rag1 $1^{--}$mice or $\operatorname{Rag} 1^{--}$mice, which were used as the tumor model, were transplanted with B16F10-OVA cells ( 0.15 million/mouse) in the groin by subcutaneous injection. For the OT1-iT-Rag $1^{--}$tumor model, Rag $I^{-}$ 1 mice were transplanted with the OT1 pro-pre-B cells transduced with Hoxb5 retroviruses (5 million/mouse) or pro-pre-B cells transduced with OT1-Hoxb5 retroviruses 6 weeks prior to the B16F10-OVA cell injection. For adoptive transfer, the splenic T cells (WT-T, OT1-T, OT1-iT) were first expanded and activated for 7 days in vitro using the Dynabeads Mouse T-Activator CD3/CD28 for T-cell expansion and activation. The expanded and activated $\mathrm{T}$ cells (10 million/mouse) were transplanted into the tumor-bearing $\mathrm{Ragl}^{-/}$mice 10 days after B16F10-OVA cell injection. The tumor size was measured every other day using calipers and calculated as length $\times$ width $\left(\mathrm{mm}^{2}\right)$. Mice with tumor sizes larger than $400 \mathrm{~mm}^{2}$ were euthanized for ethical consideration.

\section{Infiltrated 0T1-iT cell isolation and effector functional analysis} OT1-iT cells were isolated from the melanoma tumors from the tumor-bearing mice as previously described. ${ }^{23}$ The isolated cells were stained with antibodies against CD45.2, CD8a, TCRV $\alpha 2$, and TCRV 35 . For the intracellular staining, cells isolated from the tumors were first stained with the surface antibodies (CD45.2, CD11b, Gr1 and CD8), fixed, and then stained with Allophycocyanin (APC)-conjugated IFN $\gamma$ and PE-conjugated GzmB.

\section{Statistical analysis}

Flow cytometry data were analyzed by the Flowjo software. Prism7 (GraphPad) and SPSS (V.22.0) were used for the statistical analysis.

Extended experimental procedures including regents, cell culture, retrovirus preparation, and BCR analysis are described in online supplementary file.

\section{Author affiliations}

${ }^{1}$ School of Life Sciences, University of Science and Technology of China, Hefei, Anhui, China
${ }^{2}$ CAS Key Laboratory of Regenerative Biology, Guangzhou Institutes of Biomedicine and Health, Chinese Academy of Sciences, Guangzhou, Guangdong, China ${ }^{3}$ Guangdong Provincial Key Laboratory of Stem cell and Regenerative Medicine, Guangzhou Institutes of Biomedicine and Health, Chinese Academy of Sciences, Guangzhou, Guangdong, China

${ }^{4}$ University of Chinese Academy of Sciences, Beijing, China

${ }^{5}$ Department of Pediatrics, Guangzhou Women and Children's Medical Center, Guangzhou, Guangdong, China

${ }^{6}$ Joint School of Life Sciences, Guangzhou Medical University, Guangzhou, Guangdong, China

${ }^{7}$ Guangzhou Regenerative Medicine and Health-Guangdong Laboratory (GRMHGDL), Guangzhou, Guangdong, China

${ }^{8}$ Institute for Stem Cell and Regeneration, Chinese Academy of Sciences, Beijing, China

Acknowledgements We thank Penghui Zhou (SYSUCC, China) for providing 0T1 transgenic mouse.

Contributors $\mathrm{FH}, \mathrm{DH}$, and HW designed and performed the experiments, acquired and analyzed the data. JW and HW concepted and supervised this study. YL, PZ, $\mathrm{CL}, \mathrm{KW}, \mathrm{QW}$, and YG participated in multiple experiments. XL and YG performed flow cytometry (fluorescence-activated cell sorting). HW, FH, and JW contributed to the writing of the manuscript. JD, JC, and JW reviewed the manuscript, provided feedback, and all authors approved the manuscript in its final form.

Funding This work was supported by grants from the Major Research and Development Project of China (2019YFA0110203, 2019YFA0110202), CAS Key Research Program of Frontier Sciences (QYZDB-SSW-SMC057), Healthcare Cooperative Innovation Key program of Guangzhou Science and Technology Planning Project (201803040017), Strategic Priority Research Program of the Chinese Academy of Sciences (XDA16010601), Key Research \& Development Program of Guangzhou Regenerative Medicine and Health Guangdong Laboratory (2018GZR110104006), Science and Technology Planning Project of Guangdong Province (2017B030314056), and the grants from the National Natural Science Foundation of China (31600948).

Competing interests None declared.

Patient consent for publication Not required.

Ethics approval All animal experiments were approved by the Institutional Animal Care and Use Committee of Guangzhou Institutes of Biomedicine and Health, Guangzhou, China.

Provenance and peer review Not commissioned; externally peer reviewed.

Data availability statement Data are available on reasonable request. Not applicable.

Open access This is an open access article distributed in accordance with the Creative Commons Attribution 4.0 Unported (CC BY 4.0) license, which permits others to copy, redistribute, remix, transform and build upon this work for any purpose, provided the original work is properly cited, a link to the licence is given, and indication of whether changes were made. See https://creativecommons.org/ licenses/by/4.0/.

ORCID iD

Hongling Wu http://orcid.org/0000-0001-5398-294X

\section{REFERENCES}

1 Schumacher TNM. T-cell-receptor gene therapy. Nat Rev Immunol 2002;2:512-9.

2 Hinrichs CS, Rosenberg SA. Exploiting the curative potential of adoptive T-cell therapy for cancer. Immunol Rev 2014;257:56-71.

3 Marcinkowski B, Stevanović S, Helman SR, et al. Cancer targeting by TCR gene-engineered T cells directed against Kita-Kyushu lung cancer antigen-1. J Immunother Cancer 2019;7:229.

4 Richards DM, Marschall V, Billian-Frey K, et al. HERA-GITRL activates $T$ cells and promotes anti-tumor efficacy independent of FcyR-binding functionality. J Immunother Cancer 2019;7.

5 Tan Q, Zhang C, Yang W, et al. Isolation of T cell receptor specifically reactive with autologous tumour cells from tumour-infiltrating lymphocytes and construction of $T$ cell receptor engineered $T$ cells for esophageal squamous cell carcinoma. J Immunother Cancer 2019;7:232. 
6 Yang L, Baltimore D. Long-term in vivo provision of antigen-specific T cell immunity by programming hematopoietic stem cells. Proc Natl Acad Sci U S A 2005;102:4518-23.

7 Zhu Y, Smith DJ, Zhou Y, et al. Development of hematopoietic stem cell-engineered invariant natural killer T cell therapy for cancer. Cell Stem Cell 2019;25:542-57.

8 Minagawa A, Yoshikawa T, Yasukawa M, et al. Enhancing T cell receptor stability in rejuvenated iPSC-derived T cells improves their use in cancer immunotherapy. Cell Stem Cell 2018;23:850-8.

9 Themeli M, Kloss CC, Ciriello G, et al. Generation of tumor-targeted human $\mathrm{T}$ lymphocytes from induced pluripotent stem cells for cancer therapy. Nat Biotechnol 2013;31:928-33.

10 Guo R, Hu F, Weng Q, et al. Guiding T lymphopoiesis from pluripotent stem cells by defined transcription factors. Cell Res 2020;30:21-33.

11 Laiosa CV, Stadtfeld M, Xie H, et al. Reprogramming of committed T cell progenitors to macrophages and dendritic cells by C/EBP alpha and PU.1 transcription factors. Immunity 2006;25:731-44.

12 Li P, Burke S, Wang J, et al. Reprogramming of T cells to natural killer-like cells upon Bcl11b deletion. Science 2010;329:85-9.

13 Xie $\mathrm{H}$, Ye M, Feng $\mathrm{R}$, et al. Stepwise reprogramming of $\mathrm{B}$ cells into macrophages. Cell 2004;117:663-76.

14 Pires CF, Rosa FF, Kurochkin I, et al. Understanding and modulating immunity with cell reprogramming. Front Immunol 2019;10:2809.
15 Zhang M, Dong Y, Hu F, et al. Transcription factor Hoxb5 reprograms B cells into functional T lymphocytes. Nat Immunol 2018;19:279-90.

16 Weng $Q$, Hu F, Zhang M, et al. A protocol for generating induced T cells by reprogramming B cells in vivo. Cell Regen 2018;7:7-15.

17 Barker J, Verfaillie CM. A novel in vitro model of early human adult $B$ lymphopoiesis that allows proliferation of pro-B cells and differentiation to mature B lymphocytes. Leukemia 2000;14:1614-20.

18 Fournier M, Lebert-Ghali C-E, Hassawi M, et al. Paralog 4 Hox genes HOXA4 and Hoxb4 expand pro-B cells in vitro. Blood 2011;118:1299.

19 Hogquist KA, Jameson SC, Heath WR, et al. T cell receptor antagonist peptides induce positive selection. Cell 1994;76:17-27.

20 Padovan E, Casorati G, Dellabona P, et al. Expression of two T cell receptor alpha chains: dual receptor T cells. Science 1993;262:422-4.

21 Blüthmann $\mathrm{H}$, Kisielow $\mathrm{P}$, Uematsu $\mathrm{Y}$, et al. T-cell-specific deletion of T-cell receptor transgenes allows functional rearrangement of endogenous alpha- and beta-genes. Nature 1988;334:156-9.

22 Quah BJC, Parish CR. New and improved methods for measuring lymphocyte proliferation in vitro and in vivo using CFSE-like fluorescent dyes. J Immunol Methods 2012;379:1-14.

23 Yang W, Bai Y, Xiong Y, et al. Potentiating the antitumour response of CD8(+) T cells by modulating cholesterol metabolism. Nature 2016;531:651-5. 